\title{
KINETIC MODELING OF MEAT COLOR EVOLUTION DURING HEATING
}

\author{
M.M. URETA ${ }^{\dagger}$, D.F. OLIVERA ${ }^{\dagger}$, L.I. SEGURA ${ }^{\ddagger}$, V.O. SALVADORI ${ }^{\dagger *}$ and S.M. GOÑ ${ }^{\dagger *}$ \\ †Centro de Investigación y Desarrollo en Criotecnología de Alimentos, CCT-CONICET La Plata-Fac. Cs. Exactas, UNLP - \\ CICPBA. 47 y 116, B1900AJJ La Plata, Argentina. \\ + Fac. de Ingeniería, UNLP. 1 y 47, B1900TAG La Plata, Argentina. \\ smgoni@quimica.unlp.edu.ar
}

\begin{abstract}
D During cooking, both temperature and time have a large effect on physical properties of meat, mainly due to protein denaturation. This work intends to evaluate a computer vision system to measure color changes and to develop a kinetic model to predict them during meat cooking. Pieces of semitendinosus muscle were heated by immersion in a thermostatic bath at constant temperature (from 40 to $100^{\circ} \mathrm{C}$ ). Superficial color was measured using a computer vision system $(C V S)$ and a colorimeter, and represented by means of the $C I E L * a * b *$ color space. A kinetic model was developed to describe color changes during heating. The correlation coefficient between experimental and predicted values was 0.998 for colorimeter and 0.987 for $C V S$ values. In addition, the dependence with the temperature of the fitting parameters was determined. The kinetic constants present a typical Arrhenius dependence. These results encourage coupling the kinetic model to a cooking model previously developed.
\end{abstract}

Keywords--meat; color kinetics; cooking.

\section{INTRODUCTION}

Meat color is the first quality attribute that consumers perceive and is associated to freshness and safety issues (Mancini and Hunt, 2005). Besides, it is also used as an indicator of the degree of doneness (Pathare and Roskilly, 2016), although it does not consider the internal temperature, the most reliable criteria to ensure microbiological safety.

Meat cooking can be defined as the heating up to a temperature sufficiently high to denature proteins. Both temperature and cooking time have a large effect on physical properties and technological quality of meat such as texture, water content and water holding capacity, flavor development, proteins coagulation, inactivation of enzymes and color changes (Tornberg, 2005). Regarding this last one, thermal denaturation of the main proteins groups (myosin, actin, actomyosin, titin) produces an intense coagulation and a release of water, which affects the optical properties of meat (Xia et al., 2008). Therefore, during a first stage the meat becomes whiter, causing an increase of lightness ( $L^{*}$ value in the $C I E L * a * b *$ color space). This behavior has been reported in different kinds of meats: fish (Nakamura et al., 2011; Matsuda et al., 2013; Hosseinpour et al., 2013; Yu et al., 2014); pork (Lien et al., 2002); and beef (Pakula and Stamminger,
2012; Kondjoyan et al., 2014). A second stage is characterized by a decrease of $L^{*}$ values (Goñi, 2010), due to the gelatinization of collagen at prolonged heating times (Xia et al., 2008). In addition, at this stage the combination of high temperature and low water content promotes Maillard reactions (Yu et al., 2014), which confer the product desirable flavors and lead to a further decrease of $L^{*}$. Besides, the variation of red color (represented by $a^{*}$ color value) is crucial for meat products. During heating the myoglobin denaturation influences the absorption properties (AMSA, 2012; Røssvoll et al., 2014). Initially, a variation from deep red to pink (temperature $\mathrm{T}$ near $60^{\circ} \mathrm{C}$ ) is observed, later, to a greyish color ( $\mathrm{T}$ between 60 and $70{ }^{\circ} \mathrm{C}$ ), and finally, to a light brown ( $\mathrm{T}$ between 70 $80{ }^{\circ} \mathrm{C}$, Lawrie and Ledward, 2006; Kondjoyan et al., 2014). Also, the oxidation state of myoglobin has a deep impact in the color kinetics, due the so-called premature browning, where the meat looks cooked at lower temperatures than the normal ones (Hunt et al., 1999; King and Whyte, 2006).

Several authors developed kinetic models to predict color variations during meat processing. Concerning fish products, Kong et al. (2007) studied the variation of superficial color during salmon heating, pointing out two phases: a fast lightening followed by a slow browning, this last one modeled with zero order kinetics. Hosseinpour et al. (2012) described color changes during shrimp drying using zero and first order kinetics. Matsuda et al. (2013) modeled with first order kinetics the changes in superficial lightness of red sea bream during heating, dismissing the initial increase in $L^{*}$. Yu et al. (2014) followed the actin and myosin denaturation during grilling of red sea and suggested first order kinetics with temperature dependent variables. Regarding red meat, Portanguen et al. (2009) employed successive first order kinetics to predict the initial phase (lightness increase) followed by the browning stage during cooking. Pakula and Stamminger (2012) developed a method to measure color variation (using CIE XYZ space) to determine the degree of cooking. These authors indicated that lightness recording is sufficient to characterize color kinetics. Kondjoyan et al. (2014) analyzed color evolution relating it to protein denaturation. They described both consecutive stages (whitening followed by browning), and represented both of them with first order kinetics. 
On the other hand, online color measurement appears to be a very appropriate method for industrial food processes monitoring (Goñi and Salvadori, 2017). In this sense, a computer vision system has many advantages in contrast to the traditional colorimeter: very small as well as very large samples can be handled in a single measurement (Rodríguez-Pulido et al., 2012), it does not require direct contact with the sample, and it can be used remotely.

Taking into account this background, the aims of this work are to evaluate a computer vision system to measure color changes during meat cooking, and to develop a kinetic model that represents them. Thus, the kinetic model can help to predict the cooking time related to a desired degree of doneness.

\section{A. Sample preparation}

\section{METHODS}

Beef semitendinosus muscle were purchased in a local market of La Plata (Argentina), and chilled at $4{ }^{\circ} \mathrm{C}$ for 24 hrs. Slices of $6 \mathrm{~mm}$ thickness and $8.2 \pm 0.5 \mathrm{~g}$ (approx.) were packed into polyethylene bags $(60 \mu)$. Samples were cooked by immersion in a thermostatic bath (6 l capacity, Vicking Masson D, Argentina) at constant temperature (from 40 to $100{ }^{\circ} \mathrm{C}$ ). The heating times were 2, 4, 6, 8, $10,15,20,30$ and 60 minutes (at temperatures of $90{ }^{\circ} \mathrm{C}$ or higher maximum heating time was 30 minutes). Given the small thickness of the samples and the bath volume, it was assumed that the sample surface reaches almost instantaneously the bath temperature. After cooking, samples were introduced in a water-ice mixture during 30 seconds to stop the color changes. Then they were smoothly dried using absorbent paper.

\section{B. Color measurements}

Superficial color was measured using a CVS (Goñi and Salvadori, 2017), which consists on an image acquisition chamber, an illumination system, and a digital camera (Sony Alpha a3500, Japan). The samples were placed in the middle of the chamber floor, together with a X-Rite ColorChecker (X-Rite Inc., USA), which allows calibrating the $C V S$ by means of an empiric conversion model between the $R G B$ (red, green and blue) and the CIEL*a*b* color spaces. The images $(3000 \times 4000$ pixels, acquired with the same white balance) were processed using computational tools developed ad-hoc (Goñi and Salvadori, 2016). Besides, the surface color was measured with a colorimeter (Konica Minolta CR-400, Japan; D65 illuminant, $8 \mathrm{~mm}$ aperture, $2^{\circ}$ standard observer), in order to corroborate $L * a * b^{*}$ values, four measures were taken from both sides of the meat slice.

\section{Modeling of color changes}

The color of a meat sample subjected to a thermal treatment is assumed time and temperature dependent, and is expressed as a combination of two independent mechanisms (Goñi, 2010):

$$
C(t)=C^{I}(t)+C^{D}(t)
$$

where $C$ refers to each color parameter $L^{*}, a^{*}$ or $b^{*}$, and superscripts $I$ and $D$ refers to the increase and decrease mechanisms, respectively. Each mechanism is modeled independently. Taking into account published results and some preliminary tests, fractional first order variation was considered for both mechanisms, according to Eqs. (2) and (3):

$$
\begin{aligned}
& C^{I}(t)=C_{\infty}^{I}+\left(C_{0}^{I}-C_{\infty}^{I}\right)^{e^{-k} I^{t}} \\
& C^{D}(t)=C_{\infty}^{D}+\left(C_{0}^{D}-C_{\infty}^{D}\right)^{e^{-k} D^{t}}
\end{aligned}
$$

where subscripts $O$ and $\infty$ refer to the initial and the equilibrium (at prolonged times) values, and $k_{I}$ and $k_{D}$ are the kinetic constants. Color values at initial and final time are:

$$
\left\{\begin{array}{l}
C(t=0)=C_{0} \\
C(t=\infty)=C_{\infty}
\end{array}\right.
$$

Therefore, the initial and final conditions for each mechanism are established according to the set of Eq. (5); and operating on Eq. (5), Eq. (4) is obtained.

$$
\left\{\begin{array}{l}
C^{I}(t=0)=C_{0}^{I}=\alpha C_{0} \\
C^{D}(t=0)=C_{0}^{D}=(1-\alpha) C_{0} \\
C^{I}(t=\infty)=C_{\infty}^{I}=\beta C_{\infty} \\
C^{D}(t=\infty)=C_{\infty}^{D}=(1-\beta) C_{\infty}
\end{array}\right.
$$

Finally, from Eqs. (1) to (5), the kinetic model is expressed as following:

$$
\begin{aligned}
C(t)= & C_{\infty}+\left(\alpha C_{0}-\beta C_{\infty}\right) e^{-k_{I} t}+ \\
& \left((1-\alpha) C_{0}-(1-\beta) C_{\infty}\right) e^{-k_{D} t}
\end{aligned}
$$

The parameters $k_{I}, k_{D}, \alpha, \beta, C_{0}$ and $C_{\infty}$ must be obtained by fitting experimental color data. Particularly, the kinetic constants $\left(k_{I}\right.$ and $\left.k_{D}\right)$ can be related to temperature (expressed in $\mathrm{K}$ ) using the Arrhenius equation:

$$
k_{\text {Ior } D}=k_{\text {Ior } D, 0} e^{(-E a / R T)}
$$

Additionally, it has been reported that the final color $\left(C_{\infty}\right)$ strongly depends on the temperature, on the oxidation state of the meat and, in less extend, on the cooking method. In order to relate $C_{\infty}$ with temperature, the color values measured at the longest processing time at each temperature were considered.

Noteworthy that the non-linear fitting procedure can have multiple and equivalent solutions (for the same set of experimental data) relying on the starting point. In this sense, several fittings were performed to ensure a suitable solution.

\section{Statistical Analysis}

The experimental data was subjected to statistical analysis, evaluating the error between the two devices used to measure the color. In this sense, the average absolute residuals (Eq. (8)), and the total color difference $\Delta E$ (Eq. (9)) were calculated.

$$
\begin{gathered}
|\Delta C|=\frac{1}{m} \sum_{i=1}^{m}\left|C_{E, i}-C_{F, i}\right| \\
\Delta E=\frac{1}{m} \sum_{i=1}^{m} \sqrt{\left(L_{E, i}^{*}-L_{F, i}^{*}\right)^{2}+\left(a_{E, i}^{*}-a_{F, i}^{*}\right)^{2}+\left(b_{E, i}^{*}-b_{F, i}^{*}\right)^{2}}
\end{gathered}
$$

The subscripts $E$ refers to $C V S$ value and subscript $F$ refers to colorimeter value. Also, Eqs. (8) and (9) were 
used to evaluate the error of the fitting procedure. In this case, the subscripts $E$ and $F$ refer to experimental and fitted values, respectively, being the experimental values those obtained with the CVS or with the colorimeter. The subscript $i$ refers to each sampling time, and $m$ is the number of samples at each cooking temperature.

\section{RESULTS}

Figure 1 shows the variation of each color parameter at two different processing temperatures, comparing both devices. An increase in $L^{*}$ values is observed in all tests, despite the temperature.

Particularly when the treatment temperature is low (Fig. 1A) $L^{*}$ value increases slowly, reaching a constant value at prolonged times. At higher processing temperatures (Fig. 1B), the increase in $L^{*}$ is faster and after reaching a maximum, it decreases gradually, being the final value always above the initial one (raw sample). Similar behavior was reported by Pakula and Stamminger (2012) and Kondjoyan et al. (2014). Heating induces protein denaturation, causing in turn increase of structural inhomogeneities, higher scattering and increase of luminosity (Xia et al., 2008).

Regarding the parameter $a^{*}$, a decrease in all thermal treatments is recorded, down to a stable value, confirming the tendency informed by Vaudagna et al. (2002), who studied the effect of temperature in sous vide beef cooking. In other words, meat samples become less red. Higher processing temperatures cause a faster decreasing rate, in concordance with the statement of Pathare and Roskilly (2016), who found that between 50 and $80{ }^{\circ} \mathrm{C}$ the redness decreases significantly, and above $80^{\circ} \mathrm{C}$ myoglobin denatures completely. The changes on $a^{*}$ values are highly associated with the myoglobin pigment and its modifications (Xia et al., 2008).

The parameter $b^{*}$ did not present a significant variation, only a slight increase. Similar behavior of parameters $a^{*}$ and $b^{*}$ with the temperature was reported by Lien et al. (2002) but referring to pork meat.

In summary, the $L^{*}$ behavior is mainly due to structural changes, whereas $a^{*}$ and $b^{*}$ changes are more related to modifications of the chemical compounds.

Table 1 shows the color differences between both devices, calculated using Eqs. (8) and (9). A good correlation was found, except for $a^{*}$ values measured at low temperatures, where an appreciable difference was obtained, also shown in Fig. 3. As the cooking temperature increases, this difference becomes smaller, being less noticeable at the highest processing temperatures.

It is worth to mention that the $C V S$ allows measuring the color on the entire sample surface in a single measure, whereas multiple measurement points must be employed with the colorimeter. Furthermore, the sample has not uniform color, and then it is easier to obtain average color values from the $C V S$. For instance, Fig. 2 depicts the color predicted from CVS (Goñi and Salvadori, 2016) for one sample (which has been segmented to delete extreme data like fat, etc.); the minimum and maximum for each color parameter are also showed.

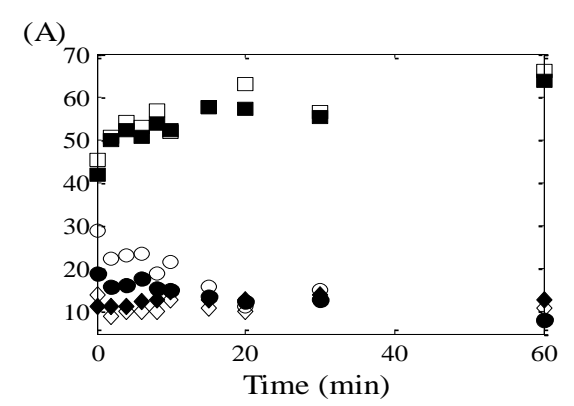

(B)

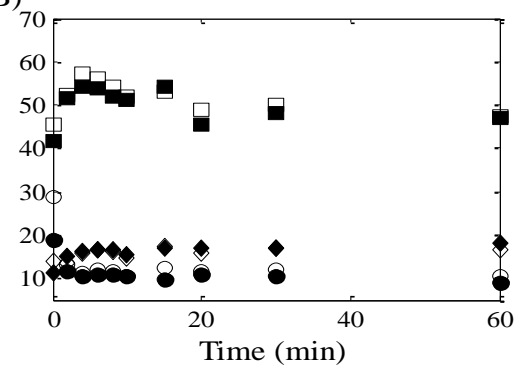

Figure 1. Experimental values of $L^{*}(\boldsymbol{\bullet}, \square), a^{*}(\bullet, \circ)$ and $b^{*}$ $(\diamond, \diamond)$ during cooking at A) $50{ }^{\circ} \mathrm{C}$ and B) $80{ }^{\circ} \mathrm{C}$. Full symbols: CVS; Empty symbols: colorimeter.

Table 1. Color differences between both devices for each treatment.

\begin{tabular}{ccccc}
\hline $\mathrm{T}\left({ }^{\circ} \mathrm{C}\right)$ & $\left|\Delta L^{*}\right|$ & $\left|\Delta a^{*}\right|$ & $\left|\Delta b^{*}\right|$ & $\Delta E$ \\
\hline 40 & $1.02 \pm 1.15$ & $6.38 \pm 2.45$ & $2.77 \pm 1.73$ & $7.31 \pm 2.44$ \\
50 & $2.15 \pm 1.73$ & $4.52 \pm 3.06$ & $2.04 \pm 0.60$ & $5.91 \pm 2.54$ \\
60 & $3.43 \pm 1.23$ & $2.44 \pm 2.83$ & $1.16 \pm 1.12$ & $4.86 \pm 2.34$ \\
70 & $2.99 \pm 1.36$ & $2.71 \pm 2.95$ & $0.97 \pm 0.70$ & $4.65 \pm 2.49$ \\
80 & $1.76 \pm 1.01$ & $2.11 \pm 2.84$ & $0.89 \pm 0.79$ & $3.21 \pm 2.75$ \\
90 & $2.94 \pm 0.85$ & $2.16 \pm 3.22$ & $0.85 \pm 0.78$ & $4.32 \pm 2.88$ \\
100 & $1.99 \pm 1.31$ & $1.93 \pm 3.08$ & $0.95 \pm 0.79$ & $3.34 \pm 3.00$ \\
\hline
\end{tabular}

Figure 3 shows the complete set of samples cooked at $40{ }^{\circ} \mathrm{C}$ and the color provided by each device (depicted as $R G B$ patches predicted from the corresponding $L * a * b *$ values). In general, $a^{*}$ value of the raw sample measured with the CVS was near 10 points higher than the one measured with the colorimeter

Figure 4 shows the graphic interface built to perform the fitting procedure. A successful fitting for each treatment was obtained.

Table 2 shows $\Delta E$ values of each processing condition, and each device. Furthermore, the correlation coefficient between the whole group of experimental and predicted values was 0.987 for the measurements with the CVS and 0.998 for the ones with the colorimeter.

Even though the individual fitting was promising, the fitting parameters $\left(k_{I}, k_{D}, \alpha, \beta\right.$ and $\left.C_{\infty}\right)$ did not present a clear dependence with the processing temperature. For this reason, a single fitting including the complete experimental dataset was performed.

First, the equilibrium values $C_{\infty}$ were fitted with temperature through simple mathematical relationships. Figure 5 shows the experimental values, measured with the $C V S$, and the result of the fitting process to characterize this dependence. Then, these equations (not shown) were included in the global fitting procedure. 

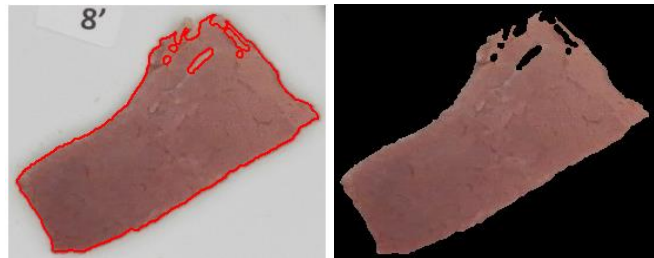

$L^{*}$

Min: 45

Max: 60

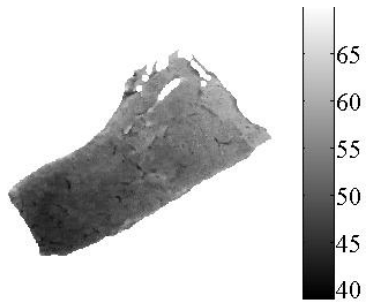

$a^{*}$

Min: 13

Max: 24

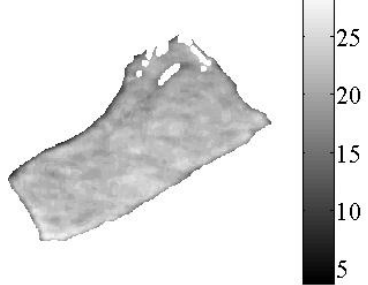

$b^{*}$

Min: 5

Max: 12

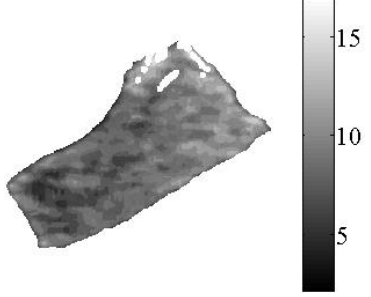

Figure 2. Experimental superficial $L^{*}, a^{*}$ and $b^{*}$ values from CVS for the sample treated 8 minutes at $50^{\circ} \mathrm{C}$.

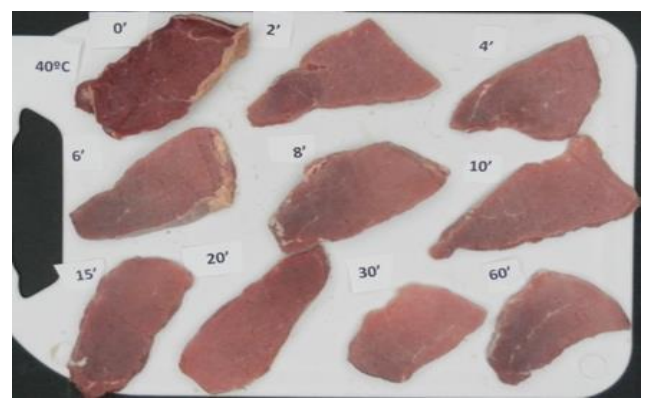

\begin{tabular}{|c|c|c|c|c|c|c|c|}
\hline \multirow{2}{*}{$t(\min )$} & \multicolumn{4}{|c|}{$C V S$} & \multicolumn{3}{|c|}{ Colorimeter } \\
\hline & $L^{*}$ & $a^{*}$ & $b^{*}$ & & $L^{*}$ & $a^{*}$ & $b^{*}$ \\
\hline 0 & 45.3 & 28.8 & 14.0 & & 41.7 & 18.8 & 11.5 \\
\hline 2 & 46.8 & 19.9 & 11.7 & & 46.2 & 16.7 & 11.9 \\
\hline 4 & 46.8 & 22.9 & 9.6 & & 46.5 & 15.4 & 14.4 \\
\hline 6 & 44.1 & 23.9 & 9.7 & & 44.0 & 21.0 & 14.0 \\
\hline 8 & 44.8 & 26.5 & 11.7 & & 45.0 & 18.2 & 15.7 \\
\hline 10 & 48.8 & 23.8 & 10.2 & & 46.9 & 16.8 & 11.5 \\
\hline 15 & 48.7 & 24.8 & 8.5 & & 48.2 & 15.8 & 11.9 \\
\hline 20 & 51.1 & 22.9 & 8.4 & & 50.4 & 16.2 & 11.5 \\
\hline 30 & 50.1 & 20.3 & 7.7 & & 50.2 & 14.9 & 12.0 \\
\hline 60 & 53.9 & 19.5 & 11.9 & & 51.8 & 15.6 & 11.9 \\
\hline
\end{tabular}

Figure 3. Color measured with both devices for cooking at 40 ${ }^{\circ} \mathrm{C}$.

Table 3 presents the results of the global fitting procedure, considering time $t$ and temperature $T$ as independent variables. For $L^{*}$ values, both mechanisms (increase and decrease) are significant, and adequately describe the experimental data. In this case, $\alpha$ and $\beta$ follow a linear tendency with the processing temperature, while the kinetic constants $k_{I}$ and $k_{D}$ present a typical Arrhenius dependence. For the other color values, $a^{*}$ and $b^{*}$, a single fractional conversion adequately represents the experimental behavior (Ec. (2)), given that including the complete model with increase and decrease terms do not improve the fitting procedure.

Table 2. Fitting errors for each experimental dataset and de-

\begin{tabular}{ccc}
\multicolumn{3}{c}{ vice. } \\
\hline $\mathrm{T}\left({ }^{\circ} \mathrm{C}\right)$ & $\Delta E_{C V S}$ & $\Delta E_{\text {Colorimeter }}$ \\
\hline 40 & $2.35 \pm 1.09$ & $1.79 \pm 1.14$ \\
50 & $2.86 \pm 1.68$ & $1.56 \pm 1.08$ \\
60 & $1.55 \pm 1.07$ & $1.33 \pm 0.88$ \\
70 & $2.70 \pm 1.49$ & $1.6 \pm 1.06$ \\
80 & $1.73 \pm 1.06$ & $1.25 \pm 1.44$ \\
90 & $1.84 \pm 2.06$ & $1.59 \pm 1.52$ \\
100 & $2.67 \pm 1.15$ & $1.20 \pm 0.97$ \\
\hline $\mathrm{t}:$ & \multicolumn{3}{c}{024681015} & & Plot data \\
$\mathrm{L}^{*} \mathrm{a}^{*} \mathrm{~b}^{*}:$ & $45.3462 .2662 .5164 .6561 .1161 .2062 .93 ; 28.78$ & Read EXCEl data..
\end{tabular}

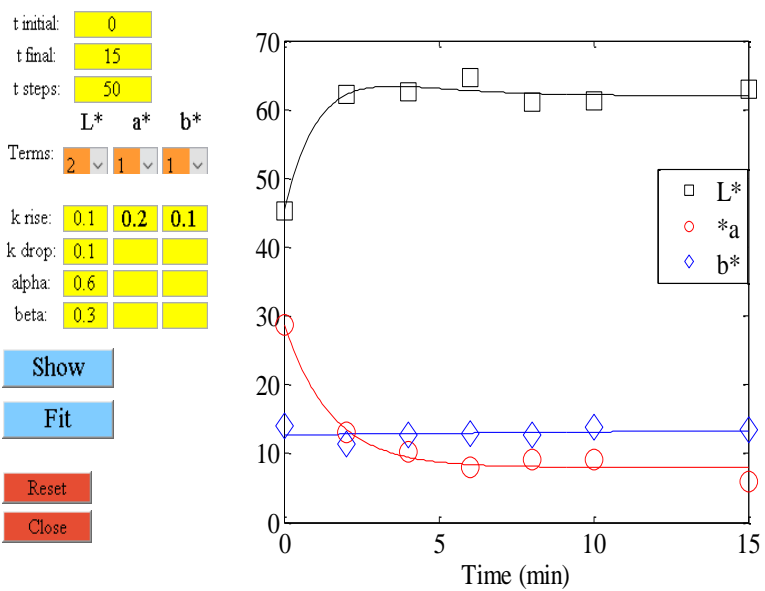

Figure 4. Graphic interface developed in MATLAB to fit the data. The data (symbols) at $70^{\circ} \mathrm{C}$ obtained with the $C V S$ is depicted. Full lines represent the fitting model.
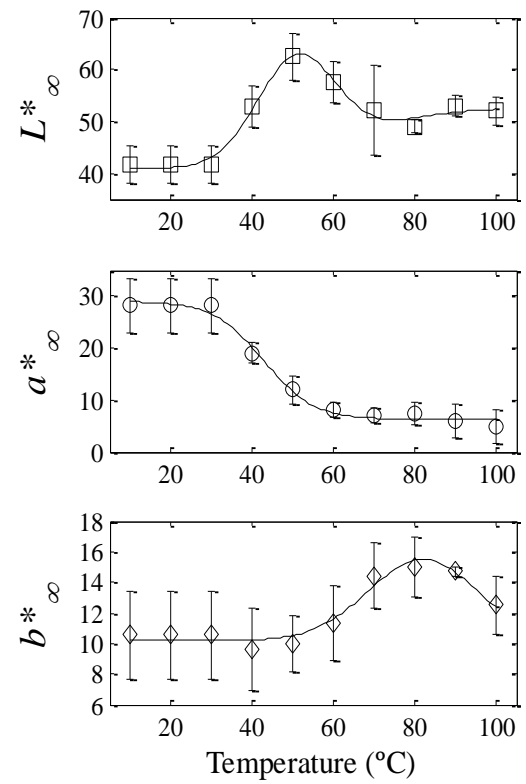

Figure 5. Equilibrium values for each color parameter $L^{*}(\square)$, $a^{*}(\diamond)$ and $b^{*}(\diamond)$. Symbols: experimental data (with $\left.C V S\right)$; full line: fitting model. 
Table 3. Fitting parameters considering the whole dataset. $\left(T:{ }^{\circ} \mathrm{C} ; E_{a}: \mathrm{kJ} / \mathrm{mol} ; k_{0}: \min ^{-1}\right)$.

\begin{tabular}{|c|c|c|c|}
\hline & $L^{*}$ & $a^{*}$ & $b^{*}$ \\
\hline \multicolumn{4}{|l|}{$C V S$} \\
\hline$C_{0}$ & 43.03 & 25.78 & 10.32 \\
\hline$\alpha$ & $0.0354+0.959 T$ & -- & -- \\
\hline$\beta$ & $0.559+0.947 T$ & -- & -- \\
\hline$E_{a, I}$ & 40.76 & 64.09 & 38.87 \\
\hline$k_{I, 0}$ & $8.0510^{5}$ & $1.5610^{9}$ & $2.1410^{5}$ \\
\hline$E_{a, D}$ & 39.38 & -- & -- \\
\hline$k_{D, 0}$ & $4.8410^{5}$ & -- & -- \\
\hline \multicolumn{4}{|c|}{ Colorimeter } \\
\hline$C_{0}$ & 42.35 & 18.24 & 11.56 \\
\hline$\alpha$ & $12.41+0.397 T$ & -- & -- \\
\hline$\beta$ & $9.99+0.362 T$ & -- & -- \\
\hline$E_{a, I}$ & 41.38 & 50.39 & 56.42 \\
\hline$k_{I, 0}$ & $1.1910^{6}$ & $6.7410^{6}$ & $1.1010^{8}$ \\
\hline$E_{a, D}$ & 34.43 & -- & -- \\
\hline$k_{D, 0}$ & $9.2410^{4}$ & -- & -- \\
\hline
\end{tabular}

(A)

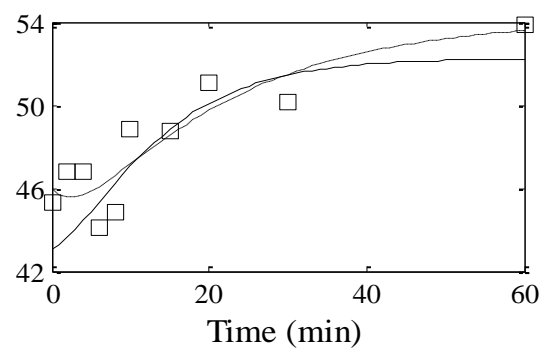

(B)

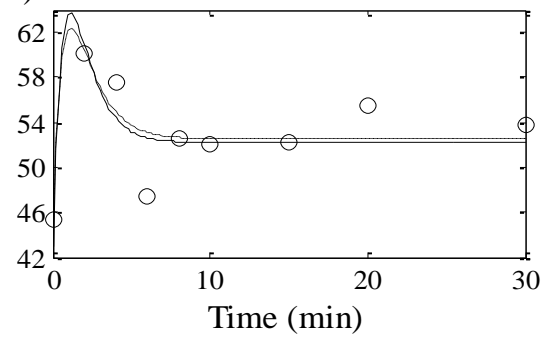

Figure 6. $L^{*}$ data at $40{ }^{\circ} \mathrm{C}(\mathrm{A})$ and $90^{\circ} \mathrm{C}(\mathrm{B})$. Symbols: experimental data; dashed line: individual fitting; continuous line: global fitting.

The kinetic model successfully represents the experimental dataset obtained with both devices. As an example, Fig. 6 compares the experimental $L^{*}$ values with those predicted with individual and global fittings.

The absolute average difference (Eq. (8)) for the CVS measurements were $1.50,1.43$ and 0.72 for $L^{*}, a^{*}$ and $b^{*}$, respectively, with $\Delta E=4.42$ in average. For data measured with the colorimeter the errors were $1.85,1.17$ and 0.55 , with a total average color difference $\Delta E=2.31$. These values were acceptable, considering that a color difference less than 2-3 cannot be detected by human eyes.

\section{CONCLUSIONS}

In general, the computer vision system provides color measurements more similar to the real color of the samples. The correlation between values measured with both devices was adequate, especially at high temperatures.
Regarding the kinetic model, it presents a good correlation with experimental data, and mainly, it was able to describe the increase-decrease behavior of $L^{*}$ values.

The global fitting allowed the prediction of color changes as a function of time-temperature, in the range of interest of cooking. In this context, these results encourage us to couple the color kinetic model with a cooking model previously developed (Goñi and Salvadori, 2010), in order to include the evolution of this quality attribute when studying the effect of different operative conditions. Thus, the cooking time could be predicted not only based on the thermal evolution inside the piece of meat, but also based on the color evolution.

\section{REFERENCES}

AMSA, Meat Color Measurement Guidelines. American Meat Science Association. http://www.meatscience. org/publications-resources/p rinted-publications/ amsa-meat-color-measurement-guidelines. Last accessed: 4/2017 (2012).

Goñi, S.M., Simulación y optimización de la cocción de productos cárneos en hornos convectivos, $\mathrm{PhD}$ Thesis, Universidad Nacional de Quilmes, Argentina (2010).

Goñi, S.M. and V.O. Salvadori. "Prediction of cooking times and weight losses during meat roasting," $J$. Food Eng., 100, 1-11 (2010).

Goñi, S.M. and V.O. Salvadori, CIELAB color measurement from digital images. https://cidca.quimica.unlp.edu.ar/colorGUI.zip. Last accessed: 12/2017 (2016)

Goñi, S.M. and V.O. Salvadori, "Color measurement: comparison of colorimeter vs. computer vision system," J. Food Meas. Charact., 11, 538-547 (2017).

Hosseinpour, S., S. Rafiee, S.S. Mohtasebi and M. Aghbashlo, "Application of computer vision technique for on-line monitoring of shrimp color changes during drying," J. Food Eng., 115, 99-114 (2013).

Hunt, M.C., O. Sørheim and E. Slinde, "Color and heat denaturation of myoglobin forms in ground beef," $J$. Food Sci., 64, 847-851 (1999).

King, N.J. and R. Whyte, "Does it look cooked? A review of factors that influence cooked meat color," J. Food Sci., 71, 31-40 (2006).

Kondjoyan, A., A. Kohler, C.E. Realini, S. Portanguen, R. Kowalski, S. Clerjon, P. Gatellier, S. Chevolleau, J.-M. Bonny and L. Debrauwer, "Towards models for the prediction of beef meat quality during cooking," Meat Sci., 97, 323-331 (2014).

Kong, F., J. Tang, B. Rasco and C. Crapo, "Kinetics of salmon quality changes during thermal processing," J. Food Eng., 83, 510-520 (2007).

Lawrie, R.A. and D.A. Ledward, "Chapter 10, The eating quality of meat," Lawrie's Meat Science. CRC Press, Boca Raton, USA. $7^{\circ}$ Ed. (2006).

Lien, R., M.C. Hunt, S. Anderson, D.H. Kropf, T.M. Loughin, M.E. Dikeman and J. Velazco, "Effects of endpoint temperature on the internal color of pork loin chops of different quality," J. Food Sci., 67, 1007-1010 (2002). 
Mancini, R.A. and M.C. Hunt, "Current research in meat color," Meat Sci., 71, 100-121 (2005).

Matsuda, H., Y. Llave, M. Fukuoka and N. Sakai, "Color changes in fish during grilling - Influences of heat transfer and heating medium on browning color," $J$. Food Eng., 116, 130-137 (2013).

Nakamura, M., W. Mao, M. Fukuoka and N. Sakai, "Analysis of the color change in fish during the grilling process," Food Sci. Tech. Res., 17, 471-478 (2011).

Pakula, C. and R. Stamminger, "Measuring changes in internal meat colour, colour lightness and colour opacity as predictors of cooking time," Meat Sci. 90, 721-727 (2012).

Pathare, P.B. and A.P. Roskilly, "Quality and energy evaluation in meat cooking," Food Eng. Rev., 8, 435-447 (2016).

Portanguen, S., A. Lebert and A. Kondjoyan, "Effect of animal diet and muscle type on the evolution of the colour of cooked beef meat", 55th International Congress of Meat Science and Technology, Copenhagen, Denmark (2009).

Rodríguez-Pulido, F.J., L. Gómez-Robledo, M. Melgosa, B. Gordillo, M.L. González-Miret and F.J. Heredia, "Ripeness estimation of grape berries and seeds by image analysis," Comput. \& Electron. Agr., 82, 128133 (2012).
Røssvoll, E., O. Sørheim, E. Heir, T. Møretrø, N.V. Olsen and S. Langsrud, "Consumer preferences, internal color and reduction of shigatoxigenic Escherichia coli in cooked hamburgers," Meat Sci., 96, 695-703 (2014).

Tornberg, E., "Effects of heat on meat proteins - Implications on structure and quality of meat products," Meat Sci., 70, 493-508 (2005).

Vaudagna, S.R, G. Sánchez, M.S Neira, E.M Insani, A.B. Picallo, M.M. Gallinger and J.A. Lasta, "Sous vide cooked beef muscles: effects of low temperaturelong time (LT-LT) treatments on their quality characteristics and storage stability," Int. J. Food Sci. \& Tech., 37, 425-441 (2002).

Xia, J., A. Weaver, D.E. Gerrard and G. Yao, "Heating induced optical property changes in beef muscle," $J$. Food Eng., 84, 75-81 (2008).

Yu, X., Y. Llave, M. Fukuoka and N. Sakai, "Estimation of color changes in fish surface at the beginning of grilling based on the degree of protein denaturation," J. Food Eng., 129, 12-20 (2014).

Received February 14, 2018.

Sent to Guest Editor February 14, 2018.

Accepted February 7, 2019.

Recommended by Guest Editor Patricia M. Hoch 